\begin{tabular}{|c|c|c|}
\hline & Int.J.Curr.Microbiol.App.Sci (2021) 10(11): $73-82$ & \\
\hline & $\begin{array}{l}\text { International Journal of Current Microbiology and Applied Sciences } \\
\text { ISSN: 2319-7706 Volume } 10 \text { Number } \mathbf{1 1}(\mathbf{2 0 2 1 )} \\
\text { Journal homepage: } \underline{\text { http://www.ijcmas.com }}\end{array}$ & $\$$ \\
\hline $\begin{array}{l}\text { EXCELLENT } \\
\text { PUBLISHERS }\end{array}$ & & \\
\hline
\end{tabular}

\title{
The Study on Different Ovulation Induction Protocols used for Intrauterine Insemination and their Pregnancy Rate in Infertile Couples
}

\author{
Yaminipriya Devarajlu ${ }^{1}$, Dhivya Venkatesan ${ }^{2}$, Balachandar Vellingiri ${ }^{2}$, \\ Ravimanickam Thangarasu $^{1 *}$ and Sarat Battina ${ }^{3}$
}

${ }^{1}$ Department of Zoology, School of Sciences, Tamil Nadu Open University, Saidapet, Chennai - 600 015, Tamil Nadu, India

${ }^{2}$ Human Molecular Cytogenetics and Stem Cell Laboratory, Department of Human Genetics and Molecular Biology, Bharathiar University, Coimbatore - 641 046, Tamil Nadu, India

${ }^{3}$ Reproductive Medicine, Indigo Women's center, Anna Nagar, chennai- 600 040, Tamil Nadu, India

*Corresponding author

\section{A B S T R A C T}

\section{Keywords}

Ovulation induction, infertility, IUI, pregnancy, HCG, HMG, Gonadotropin

\section{Article Info}

Received: 05 October 2021 Accepted: 30 October 2021 Available Online: 10 November 2021
Infertility affects millions of people of reproductive age worldwide and has an impact on their families and communities. Infertility is a disease of male or female reproductive system defined by failure to achieve a pregnancy after 12 months or more of regular unprotected sexual intercourse. Infertility is treated by different fertility drugs, ovulation induction (OI), intrauterine insemination (IUI) and in-vitro fertilization (IVF). The aim of the study is to find out the significant difference in pregnancy rate with different OI protocols used for IUI. OI is the first line treatment given to infertile women. In OI, medications are given to women for egg development and for release of eggs. OI is monitored by follicular study by ultrasonography. Semen is collected from the male partner and processed with culture media to retrieve high motile sperms, which are injected into the uterus of the female. A prospective randomized study was performed among 1343 IUI cycles. OI is started on Day 2 or Day 3 of the menstrual cycle. OI protocol is grouped according to the fertility drugs used for stimulation. The fertility drugs used for the stimulation are Clomiphene Citrate, Letrozole, Recombinant Gonadotropins, Human menopausal Gonadotropins (HMG), Human chorionic Gonadotropin (HCG). Group A uses Clomiphene citrate and HCG, Group B uses Clome, recombinant Gonadotropins and HCG. Group C uses Clome, Human menopausal Gonadotropin and HCG; Group D uses Letrozole and HCG. Group E uses Letrozole recombinant Gonadotropin and HCG. Group F used Letrozole, Human menopausal Gonadotropin and HCG. Group G used recombinant Gonadotropin and HCG. Group H used Human menopausal Gonadotropin and HCG. Group I uses only HCG. Group J was a natural cycle monitoring without any medication and was used as a control. Pregnancy rates were calculated for different age groups with different protocols. The result shows that Group B had 13\% pregnancy, Group C had 9\%, Group D had 3\%, Group E had 10\%, Group G had $11 \%$, Group G had 11\%, Group H had 7\%, Group I had 4\%, and Group J had $8 \%$ of pregnancy rate. The statistical analysis shows there is no significant difference with different stimulation protocols used. 


\section{Introduction}

Infertility is highly personal and emotional and its medical problems are growing. Infertility affects all racial and socio economic groups and because of this, every couple requires a different and comprehensive approach. Infertility is defined as a disease of the reproductive system defined by the failure to achieve a clinical pregnancy after 12 months or more of regular unprotected sexual intercourse (WHO., 2010). Infertility generates disability and thus access to health care falls under the convention on the rights of persons with disability. There are two types of infertility, namely primary infertility and secondary infertility. Primary infertility is when the woman has not conceived in her lifetime. Secondary infertility is when the woman has attained pregnancy, but might have ended in miscarriage or trying for second child (National Fertility Association., 2017).

Infertility can affect one or both partners. It is said that $30 \%$ of the infertility problems are caused by male factors, $30 \%$ caused by female factors, $30 \%$ caused by both male and female factors and the remaining $10 \%$ remains unexplained. Male infertility can be caused by obstruction of the reproductive tract causing dysfunctionalities in the ejection of sperm, hormonal disorders which leads to abnormal hormone production by the pituitary gland, hypothalamus and testicles, testicular failure to produce sperm due to varicoceles, chemotherapy and abnormal sperm function and quality. Female infertility is caused by tubal disorders (blocked fallopian tube), uterine disorders such as endometriosis, septate uterus, fibroids, disorders with ovaries - Polycystic ovarian syndrome, abnormal functioning of endocrine system where there is hormone imbalance. Some women need one or two treatments to improve fertility. Other women would need different other treatments to achieve pregnancy.
The primary goal of OI is to encourage the recruitment, maturation and development of one or two pre-ovulatory follicles. Follicle stimulating hormone (FSH) is necessary in the early phases of the cycle to recruit and select these follicles. For growth and maturation, both FSH and luteinizing hormone (LH) are necessary (Messinis and Milingos., 1997). Women who produce endogenous LH are likely to respond comparatively well to either purified FSH or preparations that contain both LH and FSH. IUI helps by placing the sperm cells directly into the uterus around the time of ovulation, making it easier for the sperm to fertilize the egg. IUI is the primary step in infertility treatment. It is very simple, less expensive and no resting period is required after the procedure (Homburg., 2003). There are a number of indications for IUI using husband semen. Ejaculatory failure is the classical indication since the male partner is unable to ejaculate into the vagina. Cervical mucus hostility is a logical indication for IUI, as it passes the mucus in the cervical canal. The most common indications for IUI are the less severe form of male factor infertility and unexplained infertility. The commonly used fertility drugs for OI are Clomiphene Citrate, Letrozole, Gonadotropin Releasing Hormone (GnRH) (Recombinant gonadotropin, Human menopausal gonadotropin (HMG), human chorionic gonadotropin (HCG)). Clomiphene citrate (Clome)is a widely used and first line drug for OI (ASRM., 2013). It acts by blocking estrogen receptors (Antiestrogenic effects). Clomiphene triggers the pituitary gland to secrete an increased amount of FSH and LH. This action stimulates the growth of ovarian follicle and thus initiates ovulation. Clomiphene citrate is started for women with anovulation, luteal phase deficiency, and unexplained infertility. Letrozole: It is a $3^{\text {rd }}$ generation triazole derivative. It is highly selective and potent. It is a non-steroidal competitive inhibitor of the aromatase enzyme system. It inhibits aromatase in ovaries and 
peripheral tissues reducing estrogen levels (Mitwally and Casper., 2000). Negative feedback being active stimulates the hypothalamus pituitary axis. Gonadotropin therapy (GnRH analogue): $\mathrm{GnRH}$ is the primary hypothalamic regulator of reproductive function. $\mathrm{GnRH}$ stimulates synthesis, storage and release of LH and FSH from gonadotropin of anterior pituitary gland. There are two types of Gonadotropin: Gonadotropin of urinary origin- Menopur 75, Gonadotropin by recombinant DNA technology (rFSH - Gonal F, rLH - Luveris, rHCG - Ovidrel).

Urinary Gonadotropin has low purity $(<5 \%)$, there is no absolute source control, cumbersome collection of urine, low specificity. Recombinant FSH has high purity, has high specific activity, and has identical amino acids sequence compared to natural FSH. HCG: Human chorionic gonadotropin (HCG) promotes the final stages of follicular maturation helping the Oocytes reach metaphase II (Polson. 1987) It is administered when the lead follicle is $>18 \mathrm{~mm}$ in diameter. It aids in final maturation and ovulation. After administering HCG, ovulation occurs within 24 hours to 48 hours later. IUI is performed before and after ovulation. The success of IUI mainly depends on the ideal sperm preparation technique which will achieve the largest number of morphologically normal motile sperm in a small volume of physiological culture media free from seminal plasma, leukocytes and bacteria (Yavas and Selub., 2004). There is no threshold of sperm concentration below which pregnancy is impossible, most conceptions occur when the number of inseminated motile sperm is 4 million or greater. There are different techniques for semen preparation mainly being swim-up and double density gradient. Our study was conducted to find out the pregnancy outcome on different stimulation protocols used for controlled OI and IUI.

\section{Materials and Methods}

\section{Study Setting and Duration}

The study is conducted at Indigo women's center, a fertility center which was established in 2016 and more than 100 IUI cycles per month were done. The data was taken from Andrology Lab - IUI register. Age group of women undergoing IUI was in the range of 20 to 45 years, information about the number of insemination cycles; medication used for OI, pregnancy was collected from January 2018 to December 2019. Informed consent was obtained from the patient for the procedure.

\section{Study Design}

A prospective randomized study performed among 1343 IUI Cycles with different OI protocols. All the stimulation cycles were started on Day $2 / 3$ of the menstrual cycle. Stimulation protocols were grouped as,

Clome + HCG, --------Group A

Clome + r Gonadotropin + HCG, -----Group B

Clome + HMG + HCG ------Group C

Letrozole + HCG,--------Group D

Letrozole $+r$ Gonadotropin + HCG,------Group E

Letrozole + HMG + HCG-------Group F

r Gonadotropin + HCG-------Group G

HMG + HCG--------Group H

only HCG----------Group I

Cycles without any fertility drugs. (No medication) --------Control 


\section{Inclusion and Exclusion criteria}

In the study, women from the age group of 1845 were included. Women who have been given fertility drugs, who have undergone single and double IUI procedures were considered in the study.

In the study, women with donor sperm insemination, no proper follicular monitoring and women who took insemination with frozen husband sperm were excluded.

OI was started on day 2 or day 3 of the women's menstrual cycle. Patients were grouped from Group A to Group I based on the fertility medication administered for OI. Follicular measurement was started from day 9 of the menstrual cycle in all the groups. When the lead follicle size was above $18 \mathrm{~mm}$ HCG trigger was given in all the groups.

Ultrasonography is carried out to confirm ovulation. $1^{\text {st }}$ IUI and ultrasonography was performed after 24 hours of HCG administration. The 2nd scan and IUI was done after 40 hours of HCG. Ultrasonography is done only to patients who have not ovulated in the first 24 hours of HCG in all the groups. If ovulation is confirmed in $1^{\text {st }}$ ultrasonography, only single IUI is done in all groups.

\section{Group A}

Patients were given Tab Clome $100 \mathrm{Mg}$ once orally from day 3 to day 7 of menstrual cycle.

\section{Group B}

Patients were given Tab Clome $100 \mathrm{Mg}$ orally from day 3 to day 7 and Injection recombinant Gonadotropin releasing hormone (Gonal F 75 IU/ Recagon 50 IU) were administered subcutaneously on alternate days (Day 3, Day 5, Day 7).

\section{Group C}

Patients were given Tab Clome $100 \mathrm{Mg}$ orally from day 3 to day 7. and Injection human menopausal Gonadotropin (HMG) (Menopur $75 \mathrm{IU}$, ) were administered subcutaneously on alternate days (Day 3, Day 5, Day 7).

\section{Group D}

Patients were given Tab Letrozole $2.5 \mathrm{Mg}$ orally twice daily from day 3 to day 7 .

\section{Group E}

Patients were given Tab Letrozole $2.5 \mathrm{Mg}$ orally twice daily from day 3 to day 7 and Injection recombinant Gonadotropin (Gonal $\mathrm{f}$ 75 IU/ Recagon 50 IU,) were administered subcutaneously on alternate days (Day 3, Day 5, Day 7).

\section{Group F}

Patients were given Tab Letrozole $2.5 \mathrm{Mg}$ orally twice daily from day 3 to day 7 . Injection human menopausal Gonadotropin (HMG) (Menopur 75IU) was administered subcutaneously on alternate days (Day 3, Day 5 , and Day 7).

\section{Group G}

Patients were administered with recombinant Gonadotropin (Gonal F 75 IU/ Recagon 50 IU,) subcutaneously on alternate days (Day 3, Day 5, Day 7).

\section{Group H}

Patients were administered Injection human menopausal Gonadotropin (HMG) (Menopur 75 IU,) on alternate days (Day 3, Day 5, Day 7). 


\section{Group I}

Patients were given only HCG triggers for ovulation.

\section{Group J}

This group is considered as a control where no fertility medicine was given. Only follicular monitoring was done and when the patient ovulated naturally IUI was performed.

\section{Follicle Measurement}

Follicle measurement was done by transvaginal ultrasonography. It is an important factor in successful OI protocol.

HCG is administered based on the follicle size measured by ultrasonography. Only after HCG administration, oocyte final maturation takes place in the dominant follicle.

Oocyte maturity is directly related to follicle size, HCG was administered when the lead follicle is $>18 \mathrm{~mm}$ in diameter. Follicle monitoring study was done either every day or on alternate days based on the size of the follicle after the 9th day of the cycle.

\section{Sperm Preparation for insemination}

The commercially available culture media was used for sperm preparation. Sil select plus is a ready to use gradient system for sperm preparation. It has 2 gradient solutions with upper layer (45\%) and lower layer (90\%).

Gradient solution contains silane coated colloidal silica particles suspended in hepes buffered EBBS (Earle's balanced salt solution) supplemented with $0.4-2.2 \mathrm{~g} / 1$ human serum albumin. Sil Select washing medium is a Hepes buffered medium which contains bicarbonate, physiological salts, glucose, lactate and human serum albumin.

\section{Procedure}

Media was equilibrated at $37^{\circ} \mathrm{C}$ for 30 minutes before the procedure.

$2.5 \mathrm{ml}$ of sil select upper layer $(45 \%)$ was transferred to sterile conical centrifuge tube.

$2.5 \mathrm{ml}$ of sil select lower layer $(90 \%)$ was placed under the upper layer.

$2.5 \mathrm{ml}$ of liquefied semen was placed above the upper layer of gradient solution.

The sample was centrifuged at 1500rpm for 10 Minutes.

During centrifugation, the sperm with good motility and morphology travel down the gradient solution and settle down at the bottom of the tube as a pellet.

After centrifugation, the supernatant was discarded.

Pellet was suspended into $3 \mathrm{ml}$ of sil select plus washing media.

Again the re-suspended pellet was centrifuged at 1500 RPM for 3 minutes.

After centrifugation, the supernatant was discarded.

Pellet was resuspended with $0.5 \mathrm{ml}$ of sil select washing media.

The sample was ready for IUI.

Semen analysis was done in raw samples and prepared samples. 


\section{IUI}

IUI is a simple procedure, where fertility specialists place the healthy sperm in the uterus at time of ovulation. A thin flexible cannula was inserted through the cervix into the uterus. Cannula was connected with $1 \mathrm{ml}$ syringe which contains a prepared sperm sample. Slowly the sperm was released into the uterus and cannula was taken out.

\section{Statistical Analysis}

Chi - square test was used to calculate the correlation between different OI protocols and their pregnancy rate. When $p<0.05$ was considered significant.

\section{Results and Discussion}

A total of 1343 cycles were grouped as Group A to Group I based on the OI protocol used for IUI. Table 1 shows the pregnancy outcome for various OI protocols. Group A, Tab Clome $100 \mathrm{mg}$ were given and 30 IUI cycles were performed, no pregnancy $(0 \%)$ is obtained. In Group B, along with Tab Clome 100mg, recombinant Gonadotropins and HCG were given and 583 IUI cycles were performed which resulted in 76 pregnancies $(13 \%)$; In Group C, Tab Clome 100mg, HMG + HCG were administered and 74 IUI cycles were performed with 7 pregnancies (9\%). In Group $\mathrm{D}$, tab Letrozole $2.5 \mathrm{mg}+\mathrm{HCG}$ were given and 29 IUI cycles was done and 1 pregnancy(3\%) obtained, in Group E Letrozole + recombinant Gonadotropins + HCG were given and 364 IUI cycles were performed with resulted in 36 pregnancies(10\%), in Group F, Letrozole + HMG + HCG were given and 19 IUI cycle was done with 2 pregnancies (11\%), in Group G, only recombinant Gonadotropins and HCG was given and 81 IUI cycles were performed which resulted in 9 pregnancies $(11 \%)$, in Group H, HMG and HCG was administered and 56 IUI cycles was performed with 4 pregnancies(7\%), in Group I only HCG was used and 68 IUI cycles was performed which obtained 3 pregnancies(4\%) and in Group $\mathrm{J}$ is used as control group, no medicines were given and 39 IUI cycles were performed which resulted with 3 pregnancies $8 \%$. The $\mathrm{p}$ value was found to be 0.27 which is greater than the 0.05 , hence the data is insignificant. Table 2 shows the data about the number of IUI cycles performed in different age groups. In the age group of 20 to 24 , there were 5 IUI cycles in Group A, group B had 54 IUI cycles, group $C$ had 6 IUI cycles, group D had 2 IUI cycles, Group E had 25 IUI cycles, Group F had 1 IUI cycle, Group G had 5 IUI cycles, group H had 12 IUI cycles, Group I had 5 IUI cycle, control group had 2 IUI cycles in this age group. In the age group of 25 to 30, there were 15 IUI cycles in Group A, group B had 304 IUI cycles, group C had 37 IUI cycles, group D had 10 IUI cycles, Group E had 189 IUI cycles, Group F had 11 IUI cycle, Group $\mathrm{G}$ had 46 IUI cycles, group $\mathrm{H}$ had 22 IUI cycles, Group I had 28 IUI cycle, control group had 16 IUI cycles in this age group. In the age group of 31 to 35 , there were 6 IUI cycles in Group A, group B had 168 IUI cycles, group $\mathrm{C}$ had 24 IUI cycles, group D had 8 IUI cycles, Group E had 109 IUI cycles, Group F had 2 IUI cycle, Group G had 19 IUI cycles, group H had 14 IUI cycles, Group I had 27 IUI cycle, control group had 19 IUI cycles in this age group. In the age group of 36 to 38 , there were 4 IUI cycles in Group A, group B had 33 IUI cycles, group C had 3 IUI cycles, group D had 8 IUI cycles, Group E had 35 IUI cycles, Group F had 4 IUI cycle, Group $\mathrm{G}$ had 8 IUI cycles, group H had 6 IUI cycles, Group I had 8 IUI cycle, control group had 2 IUI cycles in this age group. In the age group of 39 to 40, there were, 18 IUI cycles in group B, group C had 3 IUI cycles, Group E had 4 IUI cycles, Group G had 1 IUI cycles, group $\mathrm{H}$ had 1 IUI cycles, Group I had 28 IUI cycle, control group had 16 IUI cycles in this age group. 
Table.1 The following table shows the pregnancy outcome for different ovulation induction protocols

\begin{tabular}{|c|c|c|c|c|c|c|c|c|c|c|}
\hline $\begin{array}{c}\text { STIMULATION } \\
\text { PROTOCOL }\end{array}$ & $\begin{array}{c}\text { GROUP } \\
\text { A }\end{array}$ & $\begin{array}{c}\text { GROUP } \\
\text { B }\end{array}$ & GROUP & GROUP & GROUP & GROUP & GROUP & GROUP & GROUP & Control \\
\hline NO OF CYCLES & 30 & 583 & 74 & 29 & 364 & 19 & 81 & 56 & 68 & 39 \\
\hline $\begin{array}{c}\text { NO OF } \\
\text { PREGNANCIES }\end{array}$ & 0 & 76 & 7 & 1 & 36 & 2 & 9 & 4 & 3 & 3 \\
\hline PREGNANCY \% & $0 \%$ & $13 \%$ & $9 \%$ & $3 \%$ & $10 \%$ & $11 \%$ & $11 \%$ & $7 \%$ & $4 \%$ & $8 \%$ \\
\hline
\end{tabular}

Table.2 The following table shows the number of IUI cycles with different Age Group

\begin{tabular}{|c|c|c|c|c|c|c|c|c|c|c|}
\hline AGE & $\begin{array}{c}\text { GROUP } \\
\text { A }\end{array}$ & $\begin{array}{c}\text { GROUP } \\
\text { B }\end{array}$ & $\begin{array}{c}\text { GROUP } \\
\text { C }\end{array}$ & $\begin{array}{c}\text { GROUP } \\
\text { D }\end{array}$ & $\begin{array}{c}\text { GROUP } \\
\text { E }\end{array}$ & $\begin{array}{c}\text { GROUP } \\
\text { F }\end{array}$ & $\begin{array}{c}\text { GROUP } \\
\text { G }\end{array}$ & $\begin{array}{c}\text { GROUP } \\
\text { H }\end{array}$ & $\begin{array}{c}\text { GROUP } \\
\text { I }\end{array}$ & Control \\
\hline $20-24$ & 5 & 54 & 6 & 2 & 25 & 1 & 5 & 12 & 5 & 2 \\
\hline $25-30$ & 15 & 304 & 37 & 10 & 189 & 11 & 46 & 23 & 28 & 16 \\
\hline $31-35$ & 6 & 168 & 24 & 08 & 109 & 2 & 19 & 14 & 27 & 19 \\
\hline $36-38$ & 4 & 33 & 3 & 08 & 35 & 4 & 8 & 6 & 8 & 2 \\
\hline $39-40$ & & 18 & 3 & 0 & 4 & 0 & 1 & 1 & 0 & 0 \\
\hline$>40$ & & 6 & 1 & 1 & 2 & 1 & 2 & 0 & 0 & 0 \\
\hline
\end{tabular}

Table.3 The following table shows the pregnancies attained in different age groups with different protocols.

\begin{tabular}{|c|c|c|c|c|c|c|c|c|c|c|}
\hline AGE & $\begin{array}{c}\text { GROUP } \\
\text { A + VE }\end{array}$ & $\begin{array}{c}\text { GROUP } \\
\text { B +VE }\end{array}$ & $\begin{array}{c}\text { GROUP } \\
\text { C +VE }\end{array}$ & $\begin{array}{c}\text { GROUP } \\
\text { D + VE }\end{array}$ & $\begin{array}{c}\text { GROUP } \\
\text { E + VE }\end{array}$ & $\begin{array}{c}\text { GROUP } \\
\text { F + VE }\end{array}$ & $\begin{array}{c}\text { GROUP } \\
\text { G + VE }\end{array}$ & $\begin{array}{c}\text { GROUP } \\
\text { H + VE }\end{array}$ & $\begin{array}{c}\text { GROUP } \\
\text { I +VE }\end{array}$ & $\begin{array}{c}\text { Control } \\
\text { GROUP } \\
\text { J + VE }\end{array}$ \\
\hline $20-24$ & 0 & 8 & 0 & 0 & 4 & 0 & 1 & 0 & 1 & 1 \\
\hline $25-30$ & 0 & 46 & 1 & 1 & 22 & 2 & 6 & 1 & 1 & 0 \\
\hline $31-35$ & 0 & 15 & 4 & 0 & 7 & 0 & 1 & 2 & 0 & 2 \\
\hline $36-38$ & 0 & 5 & 2 & 0 & 3 & 0 & 1 & 0 & 1 & 0 \\
\hline $39-40$ & 0 & 2 & 0 & 0 & 0 & 0 & 0 & 0 & 0 & 0 \\
\hline$>40$ & 0 & 0 & 0 & 0 & 0 & 0 & 0 & 0 & 0 & 0 \\
\hline
\end{tabular}


Fig.1 Pregnancy rate in different ovulation induction protocols
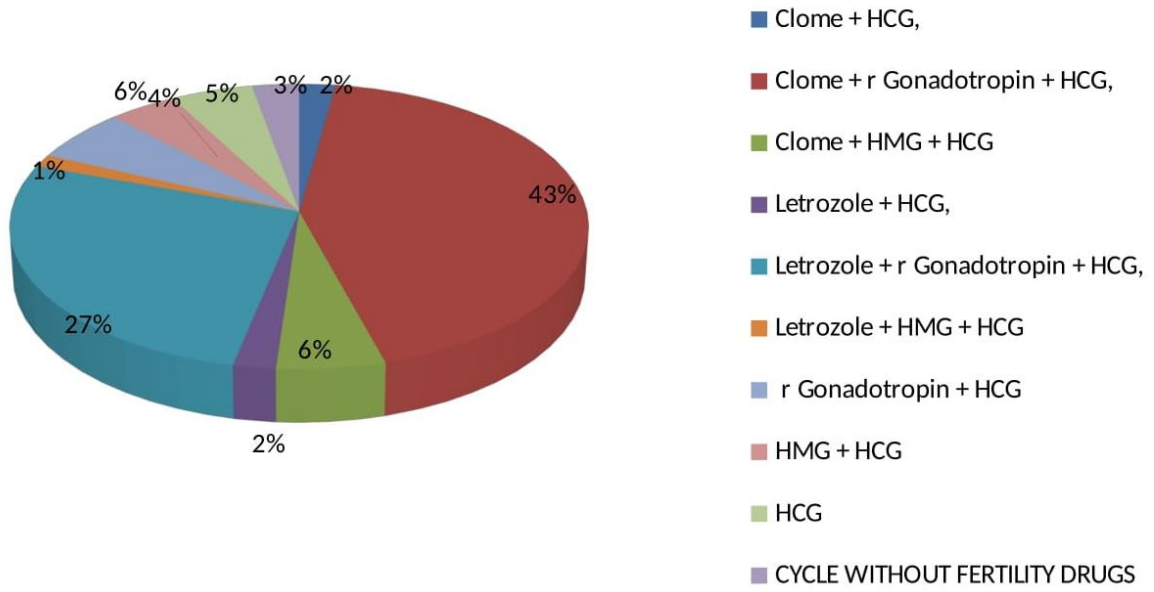

Fig.2 Data show number of cycles and pregnancy in different protocol

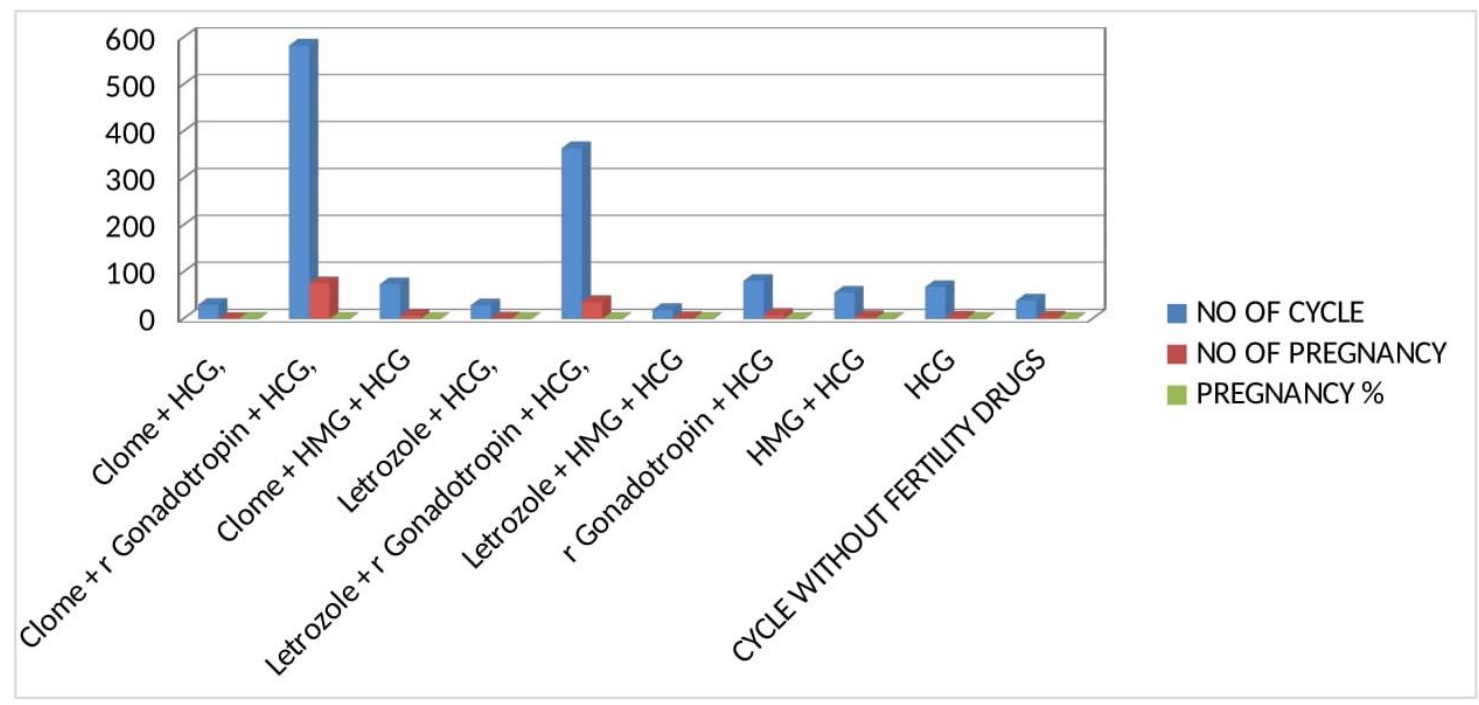

In the age group of above 40, group B had 6 IUI cycles, group C had 1 IUI cycle, Group E had 2 IUI cycles, Group F had 1 IUI cycle, Group G had 2 IUI cycles. Table 3 shows the number of pregnancies obtained in different age groups with different OI protocols. In the age group of 25 to 30 group B had 46 number of pregnancy and group $E$ had 22 number of pregnancy. In the age group of 31 to 35 , group $\mathrm{B}$ had 15 pregnancies. Figure 1 shows the percentage of pregnancy obtained in each group. Group B had the highest pregnancy compared to other groups.
IUI is the first line treatment for unexplained infertility, mild endometriosis, mild male factor infertility, and patients with poly cystic ovarian disorders. For IUI to be successful controlled $\mathrm{OI}$ and sperm preparation technique plays a vital role. When it comes to controlled OI, fertility drugs play an important role. The study was done on different fertility drugs used for OI and IUI cycles. In this study insemination cycles were grouped based on the drugs used for stimulation. In this study it was found that clomiphene citrate + recombinant gonadotropin $+\mathrm{HCG}$ had the 
highest pregnancy rate of $13 \%$. In a study conducted by Mahani and Afnan (2004) reported $\mathrm{HMG}+\mathrm{CC}$ had a higher pregnancy rate but, in our study, $\mathrm{HMG}+\mathrm{CC}$ had $9 \%$ of the pregnancy rate. Our study found that Letrozole alone has only 3\% pregnancy but Diamond et al., (2015) reported Letrozole had less cumulative pregnancy rate when compared to Clome + Gonadotropins, also another study showed Letrozole + gonadotropin had pregnancy rate of $10 \%$ which had no significant difference in pregnancy rate with Letrozole and HMG group (Baysoy et al., 2006). A study by Schorsch (2013) showed pregnancy rate per patient was significantly higher for women below the age of 25 compared to women aged 35 to 39. Our study has also reported higher pregnancy in the age group of 25 to 35 . In a study conducted by Moro et al., (2015), pregnancy rate between highly purified HMG and recombinant gonadotropin, reported there is no significant difference in pregnancy rate. In our study also the pregnancy rates between recombinant gonadotropin and $\mathrm{HMG}$ is $7 \%$ and $4 \%$ which did not give significant difference. In a study reported by $\mathrm{Xi}$ et al., (2015) Letrozole and clome combined with gonadotropin had a similar pregnancy rate. Our study Clome and gonadotropin group had $13 \%$ pregnancy compared to $10 \%$ with Letrozole and gonadotropin group. In a study comparing the efficacy of clome and Letrozole by Fauzia et al., (2013) has reported improved pregnancy results above the age of 35 . In our study Letrozole and clome did not result in any pregnancy.

The present study did not show any significant difference in different protocols used for OI. But the age of the women plays an important role, where the women with younger age had a higher chance of pregnancy. Above the age of 35 , the change of pregnancy greatly decreased in IUI cycles. IUI cycles with Clome + Gonadotropins + HCG and Letrozole +
Gonadotropin + HCG showed higher pregnancy rates when compared to the control group. To achieve higher pregnancy each patient has to be given an individualized stimulation regime to attain a higher pregnancy rate.

\section{References}

2013. Practice Committee of the American Society for Reproductive Medicine. Use of clomiphene citrate in infertile women: a committee opinion. Fertil Steril; 100:341.

Baysoy A, Serdaroglu H, Jamal H, Karatekeli E, Ozornek H, Attar E. (2006) Letrozole versus human menopausal gonadotrophin in women undergoing intrauterine insemination. Reprod Biomed Online. Aug;13(2):208-12. doi: 10.1016/s1472-6483(10)60617-7. PMID: 16895634.

Diamond M P, Legro R S, Coutifaris C, Alvero R, Robinson R D, Casson P, et al.,(2015) Letrozole, Gonadotropin, or Clomiphene for Unexplained Infertility. N Engl J Med.373:1230-40.

Fast Facts About Infertility. (2017) Available at: http://www.resolve.org/about/fastfacts-about-fertility.html. Resolve: The National Fertility Association. Accessed July 26.

Haqnawaz F, Virk S, Qadir T, Imam S, Rizvi J.(2013) Comparison of Letrozole and Clomiphene Citrate Efficacy along with Gonadotrophins in Controlled Ovarian Hyperstimulation for Intrauterine Insemination Cycles. $J$ Reprod Infertil. 14(3):138-142.

Homburg R. (2003) The case for initial treatment with intrauterine insemination as opposed to in vitro fertilization for idiopathic infertility. Hum Fertil (Camb) 6(3):122-124. doi: 10.1080/1464770312331369373. 
Mahani I M, Afnan M. (2004) The pregnancy rates with intrauterine insemination (IUI) in superovulated cycles employing different protocols (clomiphen citrate (CC), human menopausal gonadotropin (HMG) and $\mathrm{HMG}+\mathrm{CC}$ ) and in natural ovulatory cycle. J Pak Med Assoc. Oct;54(10):503-5. PMID: 15552282.

Messinis I E and Milingos S D (1997) Current and future status of ovulation induction in polycystic ovary syndrome. Hum Reprod Update 3, 235-253.

Mitwally M F, Casper R F. (2000) Aromatase Inhibition: A novel method of ovulation induction in women with polycystic ovarian syndrome. Reprod Technol.10:244-7.

Moro F, Scarinci E, Palla C, Romani F, Familiari A, Tropea A, Leoncini E, Lanzone A, Apa R.(2015) Highly purified hMG versus recombinant FSH plus recombinant $\mathrm{LH}$ in intrauterine insemination cycles in women $\geq 35$ years: a RCT. Hum Reprod. Jan;30(1):179-85. doi: 10.1093/humrep/deu302.

Polson D W, Mason H D, Saldahna M B and Franks S (1987) Ovulation of a single dominant follicle during treatment with low-dose pulsatile follicle stimulating hormone in women with polycystic ovary syndrome. Clin Endocrinol (Oxf) 26,205-212.

Schorsch M, Gomez R, Hahn T, HoelscherObermaier J, Seufert R, Skala C. (2013) Success Rate of Inseminations Dependent on Maternal Age? An Analysis of 4246 Insemination Cycles. Geburtshilfe Frauenheilkd. 73(8):808-811. doi:10.1055/s-00331350615.

World Health Organization (2010). WHO laboratory manual for the examination and processing of human semen. 5th ed. Geneva: World Health Organization; pp. xiv-271.

Xi W, Liu S, Mao H, Yang Y, Xue X, Lu X.(2015) Use of letrozole and clomiphene citrate combined with gonadotropins in clomiphene-resistant infertile women with polycystic ovary syndrome: a prospective study. Drug Des Devel Ther. 9:6001-6008. doi:10.2147/DDDT.S83259

Yavas Y, Selub M R.(2004) Intrauterine insemination (IUI) pregnancy outcome is enhanced by shorter intervals from semen collection to sperm wash, from sperm wash to IUI time, and from semen collection to IUI time. Fertil Steril. 82(6):1638-1647. doi: 10.1016/j.fertnstert.2004.04.061.

\section{How to cite this article:}

Yaminipriya Devarajlu, Dhivya Venkatesan, Balachandar Vellingiri, Ravimanickam Thangarasu and Sarat Battina. 2021. The Study on Different Ovulation Induction Protocols used for Intrauterine Insemination and their Pregnancy Rate in Infertile Couples. Int.J.Curr.Microbiol.App.Sci. 10(11): 73-82. doi: https://doi.org/10.20546/ijcmas.2021.1011.010 